\title{
Aural/Oral Performance of Children with Cochlear Implant Under Monaural and Bimodal Listening Conditions: A Parental Evaluation
}

\author{
Pooja Waghulde ${ }^{1, *}$, S. B. Rathna Kumar ${ }^{2}$, Sakeena Shora $^{3}$, Zahra Kabani \\ ${ }^{1}$ EN1 Neuro Services Private Limited, Mumbai, India \\ ${ }^{2}$ Ali Yavar Jung National Institute of Speech \& Hearing Disabilities, Mumbai, India \\ ${ }^{3}$ Bhatia Hospital, Mumbai, India
}

\section{Email address:}

poojawaghulde@gmail.com (P. Waghulde), sarathna@yahoo.co.in (S. B. R. Kumar), shora.sakeena@gmail.com (S. Shora), kabanizahra@yahoo.in (Z. Kabani)

${ }^{*}$ Corresponding author

\section{To cite this article:}

Pooja Waghulde, S. B. Rathna Kumar, Sakeena Shora, Zahra Kabani. Aural/Oral Performance of Children with Cochlear Implant Under Monaural and Bimodal Listening Conditions: A Parental Evaluation. Communication and Linguistics Studies. Vol. 5, No. 1, 2019 , pp. 8-13. doi: $10.11648 /$ j.cls.20190501.12

Received: March 24, 2019; Accepted: April 26, 2019; Published: May 20, 2019

\begin{abstract}
The study assessed the performance of children with cochlear implant (CI) under monaural and bimodal listening conditions using Parental Evaluation of Aural/Oral Performance of Children (PEACH) questionnaire. A total of 74 children using CI and their parents (either of the biological parent) served as subjects in the present study. The subjects were further divided into two groups based on mode of stimulation used in CI. Group I consisted of children using monaural stimulation in CI i.e. unilateral CI (monaural group). Group II consisted of children using bimodal stimulation in CI i.e. CI in one ear and hearing aid in opposite ear (bimodal group). The results revealed that the subjects of both groups performed significantly poorer under noisy listening conditions compared to quiet listening conditions. The subjects of both the groups performed similar under quiet listening environment. Although, both the groups performed similar under quiet listening condition, the subjects of bimodal group demonstrated significant improvement in aural/oral performance as compared to the subjects of monaural group under noisy listening environment. Thus, it can be inferred that bimodal stimulation in CI provides additional benefits as compared to monaural stimulation in $\mathrm{CI}$ especially under noisy listening environments. The findings of the present study complement the existing objective test results which have reported positive outcomes from bimodal stimulation in CI recipients.
\end{abstract}

Keywords: Cochlear Implant, Aural/Oral Performance, Monaural Stimulation, Bimodal Stimulation, Quiet Listening Environment, Noisy Listening Environment

\section{Introduction}

Cochlear implantation has become a standard procedure in the treatment of pre-lingual hearing impaired children in the last decade [1]. Cochlear implantation has dramatically changed the outcomes of children with severe-to-profound hearing loss by providing them with auditory information that is not obtainable through conventional hearing aid (HA) technology [2-4]. Children with cochlear implant (CI) are able to achieve auditory skills that exceed those of their non- implanted peers with severe-to-profound hearing loss who use HA. This is particularly true with regard to higher levels of open-set speech recognition performance [5]. However, the electrical stimulation used in CI has several limitations in comparison with acoustic amplification. The low-frequency pitch information, i.e. the fundamental frequency (F0) cues is poorly transmitted through electric stimulation [6-7]. The frequency and temporal resolutions provided by the electrode 
arrays is limited [8-9].

Providing binaural hearing is a vital goal of aural rehabilitation as binaural hearing plays an important role in understanding speech better in adverse listening conditions and localizing sound source [10]. Although binaural HA fitting has been considered a common practice, monaural stimulation has been commonly associated with CI [11]. Hence, children with monaural CI will not get the advantages of binaural hearing. Thus, monaural CI users are not only limited by their method of hearing (i.e. limitations of electrical stimulation used in CI), but also by the monaural input of sound. Bilateral input might, therefore, particularly be essential for children who tend to spend most of the day in school classrooms containing high levels of noise and long reverberation times [12].

The CI recipients have two options for bilateral stimulation, i.e. use a $\mathrm{CI}$ in each ear known as bilateral cochlear implantation or use a $\mathrm{CI}$ in one ear and a $\mathrm{HA}$ in the non-implanted ear known as bimodal hearing [13]. There has been an increase in the amount of research on the potential benefits that might arise from bilateral cochlear implantation [14-15]. Although bilateral cochlear implantation is beneficial and becoming a more common recommendation, it cannot be an option or may not be recommended for all recipients due to health issues that prevent a second surgery, financial barriers, worry about second surgery, waiting for future technology etc. [16]. Finance being the major issue particularly in developing countries, the least-expensive and non-invasive method to provide binaural hearing i.e. bimodal stimulation can be a better option [17].

Studies have reported significant speech perception improvements for bimodal hearing compared to the subjects' pre-operative bilateral HA usage as well as their postoperative usage of CI alone [18-23]. Localization abilities have also been shown to improve with bimodal hearing relative to use of either device alone [24]. The published objective test results on benefits of bimodal stimulation have been positive. Although most studies show signs of benefits from bimodal stimulation, and some studies report otherwise, there have been rare cases in which speech perception with bimodal stimulation was worse than that with CI alone [25].

The objective measures only tell us about the performance of children within the highly controlled/structured acoustic environments and does not indicate the child's performance in real-world environment [26]. The significance of utilizing subjective measures to evaluate the outcomes of HAs for young children has been well documented [27]. The necessity of including wider outcome measures, especially the parental reports while assessing the outcomes from CI in young children has been emphasized [28]. Parents spend a lot of time with their children in the everyday environment and hence their reports are often considered more reliable and representative of the child's behavioural response than assessments conducted in controlled/structured environments [29-30]. In addition, parental reports are cost-effective, facilitate professional-parent collaboration, and help parents to identify the child's strengths and needs [31].
In general, the subjective measures involve the application of auditory inventories or questionnaires to quantify parents' or caregivers' observations of their child's auditory/oral skills in everyday listening situations [32]. The Parent's Evaluation of Aural/Oral performance of Children (PEACH) developed by Ching \& Hill [33] is one such type of questionnaire designed to assess performance of children in terms of how they hear and communicate with others with their HAs and/or CI. This questionnaire has the potential as a useful clinical tool for measuring the functional outcomes of infants and children who receive HA and/or CI [33]. There is a paucity of literature assessing the benefits of bimodal stimulation among children with CI using questionnaires. Hence, the present study assessed the performance of children with CI under monaural and bimodal listening conditions using PEACH questionnaire. The findings of the present study would help to either contradict or complement the existing objective test results which have reported positive outcomes from bimodal stimulation in CI recipients.

\section{Methodology}

\subsection{Participants}

A total of 74 children using CI and their parents (either of the biological parent) served as subjects in the present study. The age of the parents ranged between 27 and 39 years with mean age of 33 years. The subjects had a minimum educational qualification of higher secondary education. The subjects who visit hospitals, special schools, government institutes and private clinics for post-surgery $\mathrm{CI}$ rehabilitation of their implanted child were recruited. The age of children with CI ranged between 5 years 6 months and 12 years 6 months with a mean age of 8 years and 9 months. The mean age of implantation of children was 2 years and 8 months, and had a mean implant age of 6 years 1 month with CI. The children were further divided into two groups based on mode of stimulation used in CI. Group I (Monaural Group): A total of 40 children using monaural stimulation in $\mathrm{CI}$ i.e. unilateral CI. The mean age of the children was 9 years 2 months. The mean age of implantation was 2 years 7 months and mean implant age was 6 years 5 months. Group II (Bimodal Group): A total of 34 children using bimodal stimulation in $\mathrm{CI}$ i.e. $\mathrm{CI}$ in one ear and HA in opposite ear. The mean age of implantation of the children was 3 years 1 month. The mean implant age was 5 years 7 months with $\mathrm{CI}$ and 4 years 3 months with bimodal stimulation.

\subsection{Tool}

The PEACH questionnaire was used as tool in the study. The PEACH questionnaire was developed as a measure of functional performance in everyday life in terms of how the child is hearing and communicating with others when using his/her HA and/or cochlear implant based on a systematic use of parents' observations. The questions focused on aural/oral behaviour in speech communication situations in real life. The questionnaire comprised 13 items, including two items 
on device usage (daily routine of use, awareness of device malfunction), one item on listening comfort (response to loud sounds), six items on listening to speech in relatively quiet situations (response to name in quiet, response to verbal instructions in quiet, follow a story read aloud, participate in conversations in quiet, recognize familiar voices, participate in conversation on a telephone), four items on listening to speech in situations that are noisy or when multiple talkers are present (response to name in noise, response to verbal instructions in noise, participate in conversations in noisy situations, participate in conversations in cars/buses/trains), and one item on awareness and recognition of environmental sounds.

\subsection{Procedure}

The PEACH questionnaire was administered on the parents either by asking them to fill up the questionnaire or by verbally interviewing them. The parents were provided with five options which were Never, Seldom, Sometimes, Often and Always. A 5-point rating scale was used for scoring these five options. The rating scale was as follows: Never $=0$, Seldom $=1$, Sometimes $=2$, Often $=3$ and Always $=4$. An item was given a score of 0 if no examples were given or if the child did not demonstrate auditory response; a score of 1 was given if one or two examples were given or the behaviour occurred $25 \%$ of the time; a score of 2 was given if three or four examples were given or the behaviour occurred $50 \%$ of the time; a score of 3 was given if five or six examples were given or the behaviour occurred $75 \%$ of the time and a score of 4 was given if more than six examples were given or the behaviour occurred more than $75 \%$ of the time. The item scores were combined into two subscale scores, one for listening in quiet (Quiet Subscale score), and one for listening in noisy environments (Noise Subscale score). Item scores were summed to derive an overall score (Overall PEACH score). The overall scores included both quiet scores and noise scores. The maximum scores obtained were 24, 20 and 44 for quiet listening condition, noisy listening condition and overall listening condition respectively. The scores were then converted into percentage and analyzed.

\section{Results and Discussion}

\subsection{Results}

The obtained data from the present study was analyzed and the results were discussed in terms of comparison of mean PEACH scores of subjects (combined monaural and bimodal groups) between quiet and noisy listening environments; comparison of mean PEACH scores between quiet and noisy listening environments among the subjects of monaural group; comparison of mean PEACH scores between of quiet and noisy listening environments among the subjects of bimodal group; comparison of mean PEACH scores between subjects of monaural and bimodal groups under quiet and noisy listening environments.

\subsubsection{Comparison of Mean PEACH Scores of Subjects (Combined Monaural and Bimodal Groups) Between Quiet and Noisy Listening Environments}

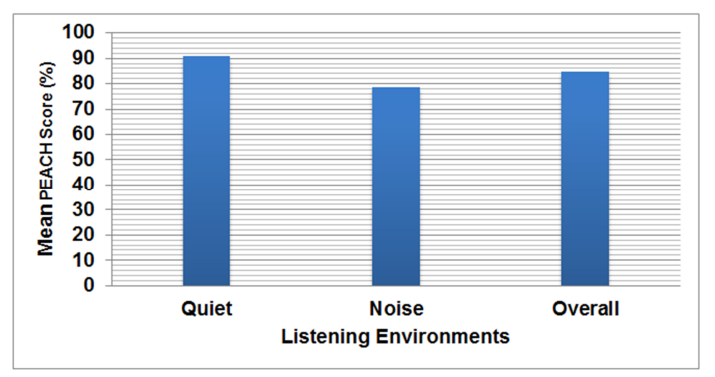

Figure 1. Comparison of mean PEACH scores of subjects (monaural and bimodal groups) between quiet and noisy listening environments.

The subjects (combined monaural and bimodal groups) obtained mean PEACH scores of $87.95 \%$ (7.76) and 70.88\% (10.45) in quiet and noisy environments respectively, and overall (combined noise and quiet subscale) mean PEACH score of $80.10 \%(8.69)$. The data were subjected one-way ANOVA in order to find out significant difference in mean PEACH scores between listening conditions. The results revealed that there was a statistically significant difference $(p<0.05)$ between mean PEACH scores for Quiet Subscale and Noise Subscale, Quiet Subscale and Overall PEACH score, and Overall PEACH score and Noise Subscale. The subjects demonstrated higher mean PEACH scores for Quiet Subscale followed by Overall PEACH score and Noise Subscale. Hence, it can be inferred that there was a significant reduction in aural-oral performance under noisy listening environment among the subjects of combined monaural and bimodal groups.

\subsubsection{Comparison of Mean PEACH Scores Between Quiet and Noisy Listening Environments Among the Subjects of Monaural Group}

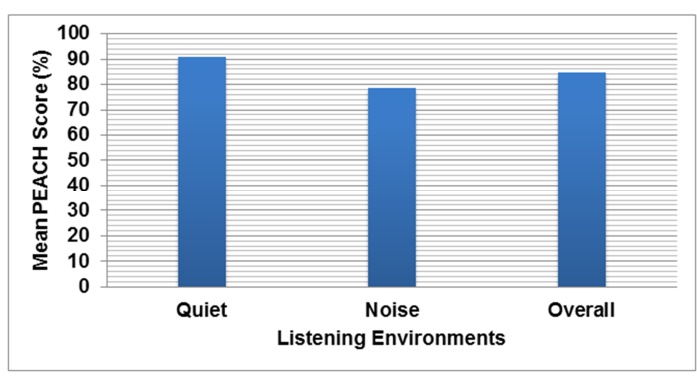

Figure 2. Comparison of mean PEACH scores between quiet and noisy listening environments among the subjects of monaural group.

The subjects of monaural group obtained a mean PEACH score of $90.27 \%(4.73)$ and $66.67 \%$ (7.27) in quiet and noisy listening environments respectively, and overall (combined noise and quiet subscale) mean PEACH score of $79.65 \%$ (5.17). The data were subjected one-way ANOVA in order to find out significant difference in mean PEACH scores between listening conditions. The results revealed that there was a statistically significant difference $(p<0.05)$ between 
mean PEACH scores for Quiet Subscale and Noise Subscale, Quiet Subscale and Overall PEACH score, and Overall PEACH score and Noise Subscale. The subjects of monaural group demonstrated higher mean PEACH score for Quiet Subscale followed by Overall PEACH score and Noise Subscale. Hence, it can be inferred that there was a significant reduction in aural-oral performance under noisy listening condition among the subjects of monaural group.

\subsubsection{Comparison of Mean PEACH Scores Between Quiet and Noisy Listening Environments Among the Subjects of Bimodal Group}

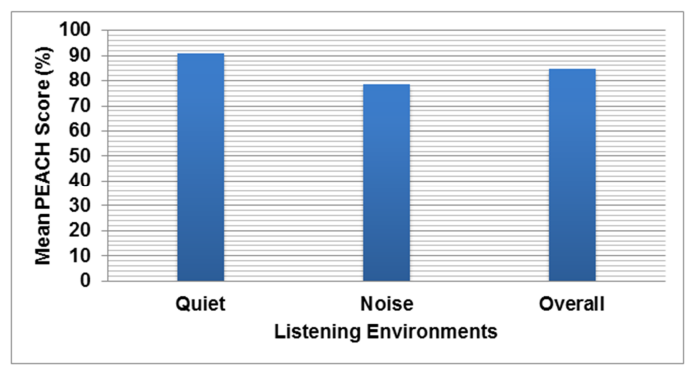

Figure 3. Comparison of mean PEACH scores between quiet and noisy listening environments among the subjects of bimodal group.

The subjects of bimodal group obtained mean PEACH scores of $90.87 \%$ (4.73) and $78.61 \%$ (4.47) in quiet and noisy environments respectively, and overall (combined noise and quiet subscale) mean PEACH score of $84.87 \%$ (3.31). The data were subjected one-way ANOVA in order to find out significant difference in mean PEACH scores between listening conditions. The results revealed that there was a statistically significant difference $(p<0.05)$ between mean PEACH scores for Quiet Subscale and Noise Subscale, Quiet Subscale and Overall PEACH score, and Overall PEACH score and Noise Subscale. The subjects of bimodal group demonstrated higher mean PEACH score for Quiet Subscale followed by Overall PEACH score and Noise Subscale. Hence, it can be inferred that there was a significant reduction in aural-oral performance under noisy listening condition among the subjects of bimodal group.

\subsubsection{Comparison of Mean PEACH Scores Between Subjects of Monaural and Bimodal Groups Under Quiet and Noisy Listening Environments}

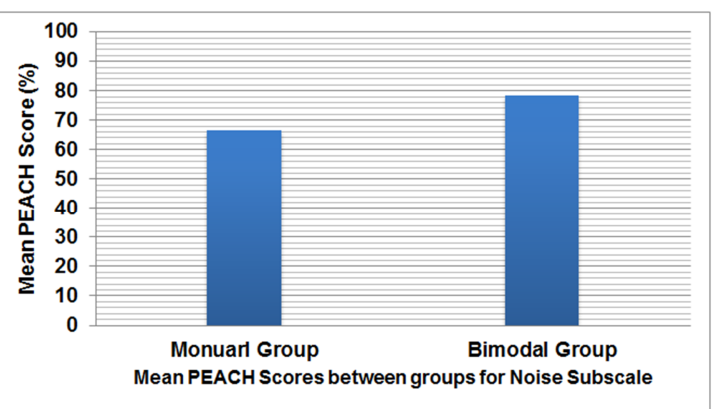

Figure 4. Comparison of mean PEACH scores between subjects of monaural and bimodal groups under quiet and noisy listening environments.
In order to compare the aural-oral performance between subjects of monaural and bimodal group, the subjects were divided into two groups based on their mode of stimulation used in CI i.e. monaural and bimodal stimulation. Group I: Consisted of a total of 40 children with CI using monaural stimulation. Group II: Consisted of a total of 34 children with CI using bimodal stimulation. The subjects of each group were further inspected in order to ensure that both the groups are matched in terms of Quiet Subscale score. This was achieved by selecting only subjects from each group who had similar Quiet Subscale score. This has resulted in the formation of two equally divided groups which were matched in terms of mean Quiet Subscale score. Group I: Consisted of 18 subjects with mean Quiet Subscale score of $90.27 \%$. Group II: Consisted of 18 subjects with mean Quiet subscale score of $90.87 \%$. The mean PEACH scores for Quiet Subscale scores of two groups were subjected to independent $\mathrm{t}$ test to assess the homogeneity between the groups in terms of Quiet Subscale score. It was found that there was no statistically significant $(p<0.05)$ difference in mean Quiet Subscale score. Hence both the groups were considered homogenous in terms of their performance in quiet environment.

The subjects of monaural and bimodal groups obtained mean PEACH scores of $66.67 \%$ (7.27) and $78.61 \%(4.47)$ under noisy listening environment. The data were subjected to independent sample $t$ test in order to find out significant difference in terms of mean PEACH scores between the subjects of monaural and bimodal groups under noisy listening environment. The results revealed that there was a statistically significant difference $(p<0.05)$ in the mean PEACH scores between the subjects of monaural and bimodal groups under noisy listening condition. The subjects of bimodal group demonstrated significantly higher performance as compared to the subjects of monaural group under noisy listening environment. Hence, it can be inferred that bimodal stimulation in $\mathrm{CI}$ provides more benefits as compared to monaural stimulation in CI especially under noisy listening environments.

\subsection{Discussion}

The present study assessed the performance of children with CI under monaural and bimodal listening conditions using PEACH questionnaire. The results revealed that the subjects of both the groups performed significantly poorer under noisy listening conditions compared to quiet listening conditions. The subjects of both the groups performed similar under quiet listening environment. Although, the subjects of both the groups performed similar under quiet listening environment, the subjects of bimodal group demonstrated a statistically significant improvement in aural/oral performance as compared to the subjects of monaural group under noisy listening environment. Thus, it can be inferred that bimodal stimulation in CI provides more benefits as compared to monaural stimulation in CI especially under noisy listening environments.

Although CI recipients achieve higher levels of speech 
recognition performance in quiet environments, the presence of background noise continues to significantly degrade speech understanding for even the best performers [34-37]. The low-frequency pitch information that aids in segregating voices through the use of fundamental frequency (F0) cues is poorly transmitted through electric stimulation as compared to acoustic hearing provided by a HA [6-7]. The frequency and temporal resolutions provided by the current electrode arrays is limited as compared to acoustic hearing provided by either HA or normal hearing [8-9].

The candidacy criteria for cochlear implantation have been relaxed to include subjects with some amount of residual hearing especially in the low-frequency region and pre-implant speech perception abilities [38]. Although HA provides limited benefit for those whose audiometric thresholds fall within the approved audiometric range for CI [16], a satisfactory access to low-frequency information can be provided through HA [39]. The low-frequency acoustic stimulation provided by the HA might allow the CI users to access the finer spectral and temporal pitch cues in the speech signal that are not well resolved by the CI [40-41]. The acoustic amplification in the low-frequencies can provide voice pitch information (F0) that assists in separating competing voices; voice onset time (VOT) cues for distinguishing voiced and voiceless consonants; and information on vowels (formant frequencies). On the other hand, the mid- and high-frequency information from the CI can provide information on manner and place of articulation of consonants. Hence, combining low-frequency acoustic hearing provided by HA compliment the mid- and high-frequency information provided by the electric hearing through CI to enhance speech intelligibility to some extent [35]. These advantages provided by low-frequency acoustic hearing therefore might lead to improved speech perception especially under noisy listening environments. The findings of the present study complement the existing objective test results which have reported positive outcomes from bimodal stimulation in CI recipients.

\section{Conclusions}

The subjects of both monaural and bimodal groups performed significantly poorer under noisy listening environment as compared to quiet listening environment. The subjects of bimodal group performed better as compared to the subjects of monaural group. Although the subjects of both the groups performed similar under quiet listening environment, the subjects of bimodal group demonstrated a statistically significant improvement in aural-oral performance as compared to the subjects of monaural group under noisy listening environment. Thus, it can be inferred that bimodal stimulation in CI provides more benefits as compared to monaural stimulation in $\mathrm{CI}$ especially under noisy listening environment.

\section{Acknowledgements}

Authors thank the participants who contributed their valuable time and unbiased support to conduct the study. We are grateful to all the parents and children for their willingness to participate, their positive attitudes and hard work. We are also thankful to Sanika Samant for her help and support.

\section{References}

[1] Baudonck, N., K. van Lierde., I. Dhooge., P. Corthals. 2011. A Comparison of Vowel Productions in Prelingually Deaf Children using Cochlear Implants, Severe Hearing-Impaired Children using Conventional Hearing Aids and NormalHearing Children. Folia Phoniatrica Logopeudics. 63: 154160.

[2] Moog J., A. Geers. 2003. Epilogue: Major Findings, Conclusions and Implications for Deaf Education. Ear \& Hearing. 24: 121S-125S.

[3] Geers A. E., J. S. Moog., J. Biedenstein., C. Brenner., H. Hayes. 2009. Spoken Language Scores of Children using Cochlear Implants Compared to Hearing Age-mates at School Entry. Journal of Deaf Studies and Deaf Education. 14: 371385 .

[4] McDermott H. J. 2011. A Technical Comparison of Digital Frequency Lowering Algorithms Available in Two Current Hearing Aids. PLoS ONE, 6 (7): e22358. doi: 10.1371/journal.pone.00223 58 .

[5] Krueger B., G. Joseph., U. Rost., A. Strauss-Schier., T. Lenarz., A. Buechner. 2008. Performance Groups in Adult Cochlear Implant Users: Speech Perception Results from 1984 until Today. Otology and Neurotology. 29 (4): 509-512.

[6] Kong Y., G. Stickney., F. Zeng. 2005. Speech and Melody Recognition in Binaurally Combined Acoustic and Electric Hearing. Journal of the Acoustic Society of America. 117: 1351- 1361.

[7] Quadrizius, S. 2008. Effects of Combined Electric and Acoustic Hearing on Speech Perception of a Paediatric Cochlear Implant User. Independent Studies and Capstones Program in Audiology and Communication Sciences, Washington: Washington University School of Medicine.

[8] Turner C. W., B. J. Gantz., M. Lowder., K. Gfeller. 2005. Benefits Seen in Acoustic Hearing plus Electric Stimulation in Same Ear. The Hearing Journal. 58 (11): 53-55.

[9] Luo X., Q. J. Fu., J. J. Galvin. 2007. Vocal Emotion Recognition by Normal-hearing Listeners and Cochlear Implant Users. Trends in Amplification. 11 (4): 301-315.

[10] Murphy, J., G. O`Donoghue. 2007. Bilateral Cochlear Implantation: An Evidence-Based Medicine Evaluation (Review). Laryngoscope. 117 (8): 1412-1418.

[11] Ching T. Y. C. 2005. The Evidence Calls for Making BinauralBimodal Fittings Routine. The Hearing Journal, 58: 32-34.

[12] Knecht, H. A., P. B. Nelson., G. M. Whitelaw., L. L. Feth. 2002. Background Noise Levels and Reverberation Times in Unoccupied Classrooms: Predictions and Measurements. American Journal of Audiology, 11 (2): 65-71.

[13] Potts L., M. Skinner., R. Litovsky., M. Strube., F. Kuk. 2009. Recognition and Localization of Speech by Adult Cochlear Implant Recipients Wearing a Digital Hearing Aid in the Nonimplanted Ear (Bimodal Hearing). Journal of the American Academy of Audiology, 20 (6): 353-373. 
[14] Zeitler D. M., M. A. Kessler., V. Terushkin., T. J. Roland., M. A. Svirsky., A. K. Lalwani., S. B. Waltzman. 2008. Speech Perception Benefits of Sequential Bilateral Cochlear Implantation in Children and Adults: A Retrospective Analysis. Otology \& Neurotology. 29 (3): 314-325.

[15] Van Deun. L., A. van Wieringen., T. Francart., F. Scherf., I. J. Dhooge., N. Deggouj., C. Desloovere., P. H. van de Heyning., F. E. Offeciers., L. de Raeve., J. Wouters. 2010. Bilateral Cochlear Implants in Children: Binaural Unmasking. Audiology \& Neurotology. 14: 240-247.

[16] Huart S. A., C. S. Sammeth. 2008. Hearing Aids plus Cochlear Implants: Optimizing the Bimodal Paediatric Fitting, The Hearing Journal. 61 (11): 54-48.

[17] Kumar S. B. R., P. Mohanty., S. G. R. Prakash. 2010. Speech Recognition Performance in Children with Cochlear Implant using Bimodal Stimulation, Indian Journal of Otolaryngology and Head Neck Surgery. 62 (4): 342-345.

[18] Ching T. Y. C., C. Psarros., M. Hill., H. Dillon., P. Incerti. 2001. Should Children who use Cochlear Implants Wear Hearing Aids in the Opposite Ear? Ear and Hearing. 22: 365380 .

[19] Hamzavi J., S. M. Pok., W. Gstoettner., W. D. Baumgartner. 2004. Speech Perception with a Cochlear Implant used in Conjunction with a Hearing Aid in the Opposite Ear. International Journal of Audiology. 43 (2): 61-65.

[20] Ching T. Y. C., E. van Wanrooy., M. Hill., H. Dillon. 2005. Binaural Redundancy and Inter-aural Time Difference Cues for Patients Wearing a Cochlear Implant and a Hearing Aid in Opposite Ears. International Journal of Audiology. 44 (9): 513-521.

[21] Morera C., M. Manrique., L. Ramos., L. Garcia-Ibanex., L. Cavalle., A Huarte., et al. 2005. Advantages of Binaural Hearing Provided through Bimodal Stimulation via a Cochlear Implant and a Conventional Hearing Aid: A 6-month Comparative Study. Acta Oto-Laryngologica. 125 (6): 596606.

[22] Gifford R. H., M. F. Dorman., S. A. McKarns., A. T. Spahr. 2007. Combined Electric and Contralateral Acoustic Hearing: Word and Sentence Intelligibility with Bimodal Hearing. Journal of Speech, Language, and Hearing Research. 50: 835843.

[23] Kumar S. B. R., P. Mohanty. 2016. Benefits of Bimodal Stimulation in Children with Cochlear Implant: Role of Contralateral Residual Acoustic Hearing and Auditory Experience with Bimodal Stimulation. International Journal of Allied Medical Science and Clinical Research. 4 (1): 136147.

[24] Seeber B. U., U. Baumann., H. Fastl. 2004. Localization ability with Bimodal Hearing Aids and Bilateral Cochlear Implants. Journal of the Acoustical Society of America. 116 (3): 1698-1709.

[25] Zhang T. 2008. The Benefits of Acoustic Input to Combined Electric and Contralateral Acoustic Hearing. Doctoral Dissertation. College Park: University of Maryland.

[26] Bagatto M. P., S. T. Moodie., R. C. Seewald., D. J. Bartlett., S. D. Scollie. 2011. A Critical Review of Audiological Outcome Measures for Infants and Children. Trends in Amplification. 15 (1-2): 23-33.
[27] Arlinger S. D. 2001. How to Assess Outcome of Hearing Aid Fitting in Children? Scandinavian Audiology. 30 (2): 68-72.

[28] Lin F. R., N. Y. Wang., N. E. Fink., A. L. Quittner., L. S. Eisenberg., E. A. Tobey. 2008. CDaCI Investigative Team Assessing the use of Speech and Language Measures in Relation to Parental Perceptions of Development after Early Cochlear Implantation. Otology \& Neurotology. 29(2): 208.

[29] Dale P. 1991. The Validity of a Parent Report Measure on Vocabulary and Syntax at 24 Months. Journal of Speech, Hearing Research. 34: 565 -571.

[30] Boudreau D. 2005. Use of a Parent Questionnaire in Emergent and Early Literacy Assessment of Preschool Children. Language, Speech, Hearing Services in Schools. 36: 33-47.

[31] Crais E. 1995. Expanding the Repertoire of Tools and Techniques for Assessing the Communication Skills of Infants and Toddlers. American Journal of Speech Language Pathology. 4 (3): 47-59.

[32] Quar T. K., T. Y. C. Ching., S. Z. Mukari., P. Newall. 2012. Parents' Evaluation of Aural/Oral Performance of Children (PEACH) Scale in the Malay Language: Data for NormalHearing Children. International Journal of Audiology. 51 (4): 326-333.

[33] Ching T. Y. C., M. Hill. 2007. The Parents' Evaluation of Aural/Oral Performance of Children (PEACH) Scale: Normative Data. Journal of American Academy of Audiology. 18: 220-235.

[34] Nascimento L. T., M. C. Bevilaqua. 2005. Evaluation of Speech Perception in Noise in Cochlear Implanted Adults, Brazilian Journal of Otorhinolaryngology. 71 (4): 432-438.

[35] Ching T. Y. C., E. van Wanrooy., H. Dillon. 2007. BinauralBimodal Fitting or Bilateral Implantation for Managing Severe to Profound Deafness: A Review, Trends in Amplification. 11: 161-192.

[36] Cullington H. E., F. G. Zeng. 2010. Bimodal Hearing Benefit for Speech Recognition with Competing Voice in Cochlear Implant Subject with Normal Hearing in Contra Lateral Ear. Ear and Hearing. 31 (1): 70-73.

[37] Gifford, R. H., A. P. Olund., M. Dejong. Improving Speech Perception in Noise for Children with Cochlear Implants. Journal of American Academy of Audiology. 22 (9): 623-632.

[38] Fitzpatrick, E. M., C. Seguin., D. Schramm., J. Chenier., S. C. Armstrong. 2009. Users' Experience of a Cochlear Implant Combined with a Hearing Aid. International Journal of Audiology. 48: 172-182.

[39] Boothroyd A. 2008. The Acoustic Speech Signal. In: J. R. Madel., C. Flexer (eds.), Paediatric Audiology (pp. 159-167). NY: Thieme.

[40] Holt, R. F., K. I. Kirk., L. S. Eisenberg., A. S. Martinez., W. Campbell. Spoken Word Recognition Development in Children with Residual Hearing Using Cochlear Implants and Hearing Aids in Opposite Ears. Ear and Hearing, 26: 82-91.

[41] Henry B. A., C. W. Turner. 2003. The Resolution of Complex Spectral Patterns by Cochlear Implant and Normal Hearing Listeners. Journal of the Acoustical Society of America. 113: 2861-2873. 